\title{
Association of PV, gas micro turbine and short term storage system to participate in frequency control
}

\author{
HERBRETEAU J. ${ }^{1,4}$, COURTECUISSE V.${ }^{1,2}$, PENG L. ${ }^{1,3}$, DEGOBERT Ph. ${ }^{1,4}$,ROBYNS B. ${ }^{1,2}$, \\ and FRANCOIS B. ${ }^{1,3}$ \\ Laboratoire d'Electrotechnique et d'Electronique de Puissance de Lille (L2EP) ${ }^{1}$ \\ Ecole des Hautes Etudes d'Ingénieur (HEI), 13 rue de Toul, F-59046, Lille, France ${ }^{2}$ \\ Ecole Centrale de Lille, Cité Scientifique, BP48, 59651 Villeneuve d'Ascq, France ${ }^{3}$ \\ Ecole Nationale Supérieure des Arts et Métiers, 8 rue Camille Guérin, 59200 Lille, France ${ }^{4}$
}

\begin{abstract}
The goal of this work is to propose a supervision methodology for hybrid generation systems. This strategy must improve the grid insertion of renewable distributed generator (DG) by associating them with a short term storage and available decentralized generator (ADG). A fuzzy logic supervisor (FLS) is proposed to maintain a reference power and to contribute to frequency control with a hybrid generator $(\mathrm{HG})$ based on photovoltaic arrays, gas micro turbine and supercapacitors. A detailed method to build this supervisor is proposed. The proposed fuzzy logic supervisor is compared with a basic supervision strategy The FLS gives a better storage management by reducing high and low saturation state, thus the HG can provide a more regular power reserve to contribute to the primary frequency control. The performance of this supervisor is shown with the help of simulations.
\end{abstract}

\section{Keywords}

PV generator, gas micro turbine, supercapacitor, frequency control, distributed generation, fuzzy logic supervisor.

\section{Introduction}

The electricity market liberalization and the development of the decentralized generation lead to many new scientific and technical issues. The major problem, which is experienced with decentralized energy sources, and particularly renewable energy ones, is their limited abilities for contributing effectively to the power system management [2], [3].

The Micro grid concept with an association of DG and storage units appears as a cluster of loads and microsources operating as a single controllable system that can provide both power and heat to its local area. A Micro grid must carry out an optimal use of local distributed resources, feeding of local loads and simplicity of operation [1].

This paper focuses particularly on the participation of $\mathrm{PV}$ power station and a available dispersed generating (ADG) system, a gas micro turbine (GMT), associated with supercapacitors as a short term storage unit (SC) to the frequency control. Some dispersed generator (DG) technologies use back to back power electronic converters for the grid connection. The intermediate DC voltage creates an electrical decoupling between the machine and the grid. Consequently there is no natural link between the network frequency and the rotational speed of the generator, but this link can be introduced artificially in the control strategy of the network side converter; papers [4] and [5] investigate this possibility.
The aim of this paper is to describe a supervision strategy for hybrid generation systems connected to the grid in order to improve the grid integration of dispersed generators. A methodology based on fuzzy logic [6] is presented to build the supervisor. The strategy must contribute to the frequency control thanks to a droop characteristic [7]. A best smoothing of the active power allows to improve the frequency stability. The proposed fuzzy logic supervisor is compared with a basic supervision strategy [8]. The FLS gives a better storage management by reducing high and low saturation state, thus the HG can provide a more regular power reserve to contribute to the primary frequency control.

In section II the system and its modelling are described. Section III makes a short review of frequency control. Section IV explains a basic supervision strategy. In section $\mathrm{V}$ a fuzzy logic based supervision strategy is developed. The performance of the proposed strategies is shown with the help of simulations with Matlab Simulink $®$. These results are discussed in section VI.

\section{Modelling of the system under study}

To be able to generate a reference power and to ensure a power reserve, the studied generating system includes PV generators, a short term storage unit and a gas micro turbine. This hybrid generating system is connected at one point of a simple network (Fig.1) and must be seen from the network manager as a classical source.

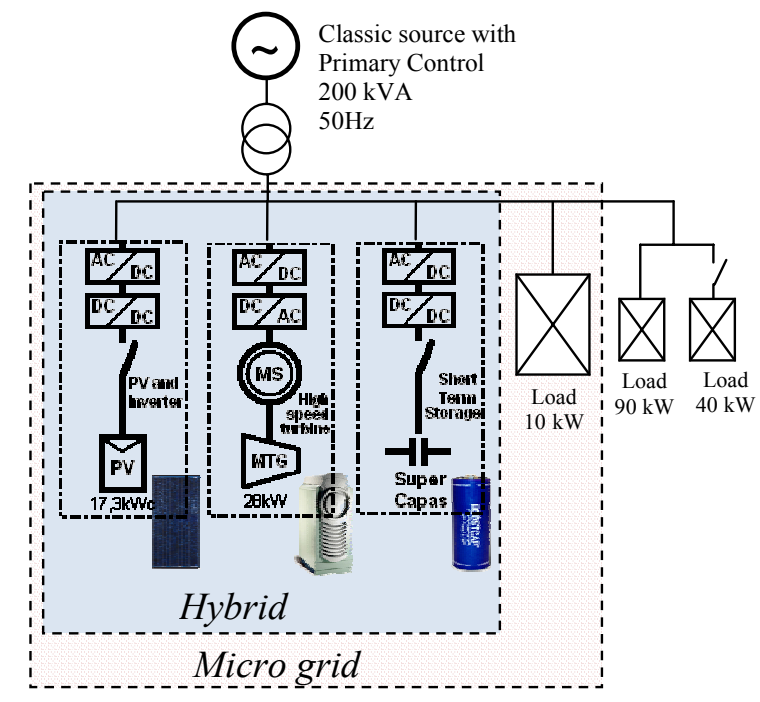

Fig. 1: System studied 


\section{A. Photovoltaic array $(P V)$}

The used timing PV characteristic has been measured at the ENSAM Engineering School in Lille (France) installation on May 2006 [8]. The PV system uses the Maximum Power Point Tracking. The sampled step is $1 \mathrm{~s}$. The installed PV peak power is $17.3 \mathrm{~kW}$.

\section{B. Gas Micro Turbine (GMT)}

A first order transfer function is used to model the GMT [9]. Table 1 presents the identified rise time value of the output power. For instance, the GMT has a 40s time response for a $10 \mathrm{~kW}$ positive variation with a $15 \mathrm{~kW}$ initial power. This model is accurate for a grid connected system mode. The GMT is a $28 \mathrm{kWelectric}$ Capstone micro turbine. The minimum electrical power is fixed at $14 \mathrm{~kW}$ to avoid an efficiency decreasing due to the heat. An assumption is that the GMT cogeneration is not used.

\begin{tabular}{|c|c|c|c|c|c|c|}
\hline$\Delta \mathbf{P} / \mathbf{P}$ & $\mathbf{5 0 0 0}$ & $\mathbf{1 0 0 0 0}$ & $\mathbf{1 5 0 0 0}$ & $\mathbf{2 0 0 0 0}$ & $\mathbf{2 5 0 0 0}$ & $\mathbf{2 8 0 0 0}$ \\
\hline $\mathbf{- 2 5 0 0 0}$ & 50 & 50 & 50 & 50 & 50 & 50 \\
\hline $\mathbf{- 1 5 0 0 0}$ & 52 & 52 & 52 & 39 & 26 & 18 \\
\hline $\mathbf{- 1 0 0 0 0}$ & 44 & 44 & 28 & 12 & 15 & 16 \\
\hline $\mathbf{- 5 0 0 0}$ & 36 & 28 & 22 & 12 & 12 & 10 \\
\hline $\mathbf{0}$ & 37 & 25 & 24 & 15 & 13 & 9 \\
\hline $\mathbf{5 0 0 0}$ & 38 & 22 & 26 & 18 & 14 & 8 \\
\hline $\mathbf{1 0 0 0 0}$ & 38 & 38 & 40 & 42 & 23 & 12 \\
\hline $\mathbf{2 0 0 0 0}$ & 38 & 38 & 38 & 30 & 21 & 16 \\
\hline $\mathbf{2 5 0 0 0}$ & 49 & 49 & 49 & 49 & 49 & 49 \\
\hline
\end{tabular}

Tab. 1: GMT rise time value (s) of output power (W)

\section{Supercapacitors (SC)}

Supercapacitors are represented by a first order transfer function [5]. A $16.5 \mathrm{~F} / 480 \mathrm{~V}$ Maxwell supercapacitor bank with a power varying from -66 to $66 \mathrm{~kW}$ and a specific energy of $138.6 \mathrm{kWh} / \mathrm{Kg}$ is used.

\section{Frequency control}

In an inter-connected network, the adjustment of the frequency is divided into three types which have different temporal aims:

- The aim of the primary control is to balance the production, with the consumption by keeping the frequency in a variation domain (fixed by the network operator).

- The secondary control brings back the frequency to its set point and restores the power exchanges within boundaries.

- The tertiary control adapts the production plan of the various groups to manage an available power reserve in an economic way with a security margin.

This paper focuses on the primary and secondary control. With synchronous generators it is carried out by regulating the alternator speed. The participation of a power station in the primary control is defined by its linear relation called droop line shown in Fig. 2. Characteristics of this line are its slope and the difference between the nominal output power of the group and the maximum capacity, which is called the primary power reserve. The quantity of this primary power reserve is defined by the network manager according to technical and economical criteria. The secondary control is a signal managed and sent by the grid manager [10],[11].

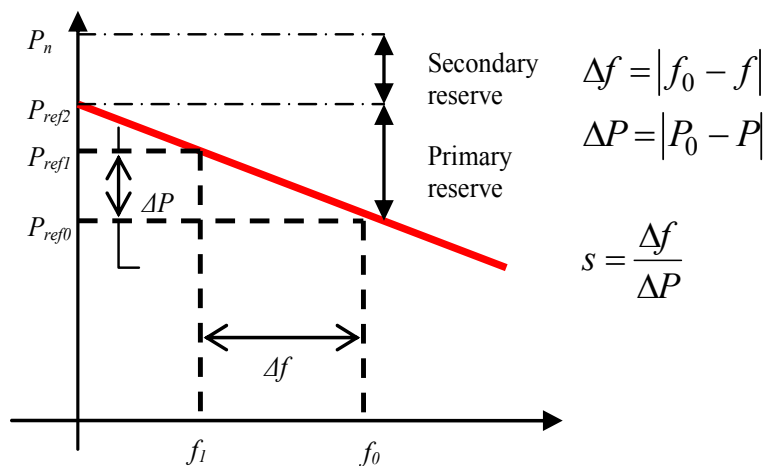

Fig. 2: Idealised governor characteristic of a turbo alternator

\section{Basic supervision strategy}

A basic supervision strategy has been developed [8] in order to achieve a smoothing power with the hybrid system shown in Fig.1. A power model is presented in Fig.3. The input power is the fluctuating photovoltaic power $\left(\mathrm{P}_{\text {pvmes }}\right)$. The reference power of the supercapacitors enables to smooth the fast fluctuations of the power thanks to a high-pass filter. Then the smoothed power is compared to the reference power and the gas micro turbine produces the missing power $\left(\mathrm{P}_{\mathrm{GMTref}}\right)$. $\mathrm{A}$ feedback-loop with a proportional controller improves the GMT response.

The proposed fuzzy logic supervisor will be compared to this basic strategy.

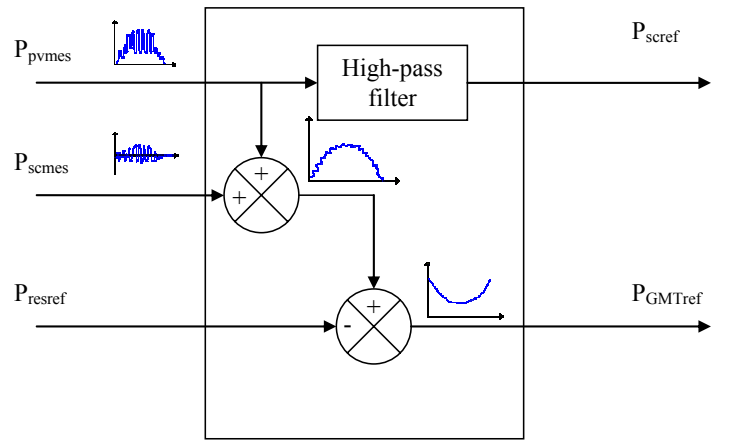

Fig3. Basic supervision strategy

\section{Fuzzy logic based supervision strategy}

The aim of this paper is to propose a supervision strategy of the hybrid system, shown in Fig.1, to follow a reference power (determined for instance by the weather forecast, market laws, network manager constraints, etc.) and to ensure primary and secondary frequency control.

\section{A. System under study}

This supervisor (Fig.4) must provide the reference power which is fixed by the network manager and maintain a primary power reserve.

Due to the complexity of the system, the random behaviour of the PV irradiance and to the network load, the fuzzy logic is well adapted to develop the supervisor 
[12] and [13]. The hybrid system behaviour must be similar to the one of a traditional source. The storage size and the power of the ADG are fixed a priori; their sizing will not be studied in this paper.

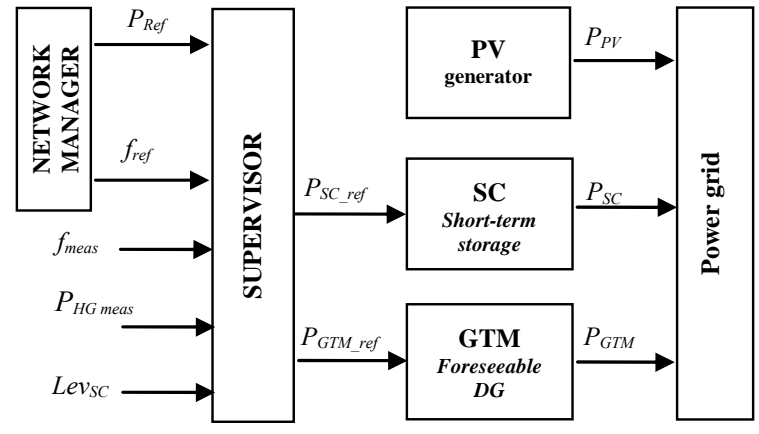

Fig.4 Supervisor of the system under study

Inputs of the supervisor are:

- The reference power $P_{r e f}$ and the reference frequency $f_{\text {ref. }}$.

- The sensed frequency $f_{\text {meas }}$, of the hybrid generators power $P_{H G m e a s}$ and of the SC storage level $L e v_{S C}$.

In the hybrid System SC have to filter the fast fluctuations of the generated power. GMT has to smooth the power and to avoid the SC saturation state level.

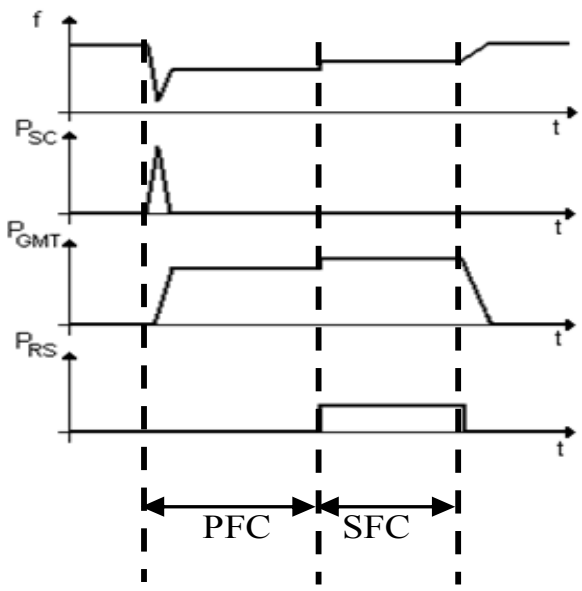

Fig. 5 Frequency control

For the primary frequency control, SC is used to decrease a peak of frequency during some seconds thanks to this fast rise time. After the GMT performs the primary frequency control (PFC) automatically and the secondary frequency control (SFC) thanks to an order from the grid

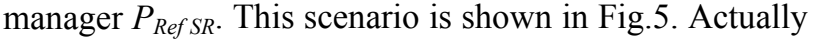
$P_{\text {Ref SR }}$ is a signal called secondary load frequency level, comprised between -1 and +1 , which is send to the generation sets in order to change their power set point values.

\section{B. Structure of the supervisor}

The structure of the supervisor shown in Fig.6 includes two parts:

- A fuzzy logic supervisor, which aims is to provide the reference power and to allow to participate in the primary frequency control.

- A droop characteristic, which performs the primary frequency control.
The inputs of the supervisor are the fast fluctuations of the power $\Delta P p v_{\text {filter }}$, the SC level Lev $\mathrm{SC}_{\mathrm{SC}}$, the variation of frequency $\Delta f$ and the power $\Delta P$ with $\Delta P=P^{\prime}{ }_{\text {Ref }}-P_{H G \text { meas }}$. The reference power of the hybrid generator is the sum of three terms (1):

$$
P_{\text {Ref }}^{\prime}=P_{\text {Ref }}+P_{\text {Ref PR }}+P_{\text {Ref } S R}
$$

with $P_{R e f P R}$ the power reference for the primary control and $P_{R e f S R}$ for the secondary control.

Filter 1 and filter 2 allow to keep only the fast fluctuations of power or frequency.

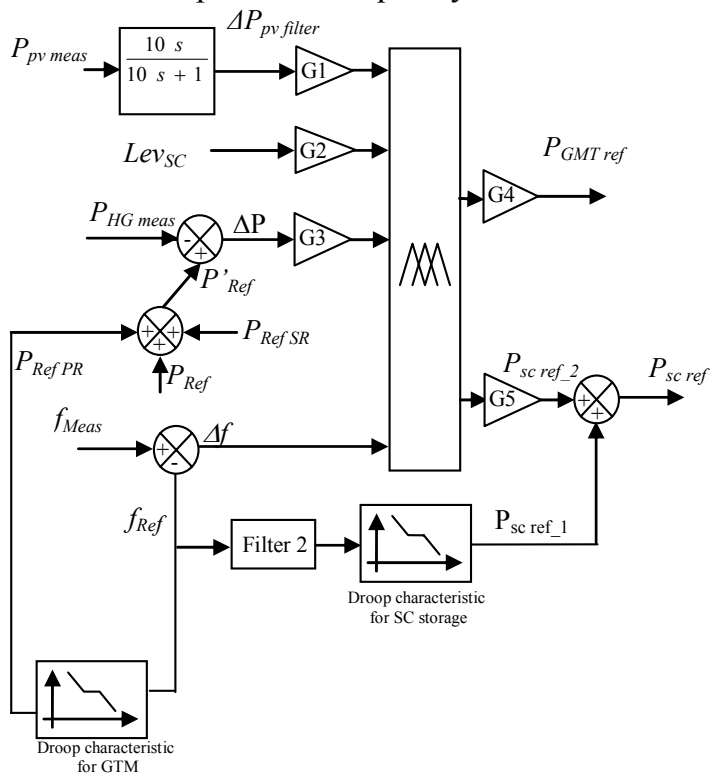

Fig.6 Structure of the supervisor

The SC and GMT reference power are respectively the sum of the power references for the frequency control $P_{\text {Sc }}$ ref $1_{1}$ [resp. P PMTref I] and the smoothing of power PSc $_{\text {ref_2 [resp. P }}$ GMTref_2].

\section{Chart representation of operating modes}

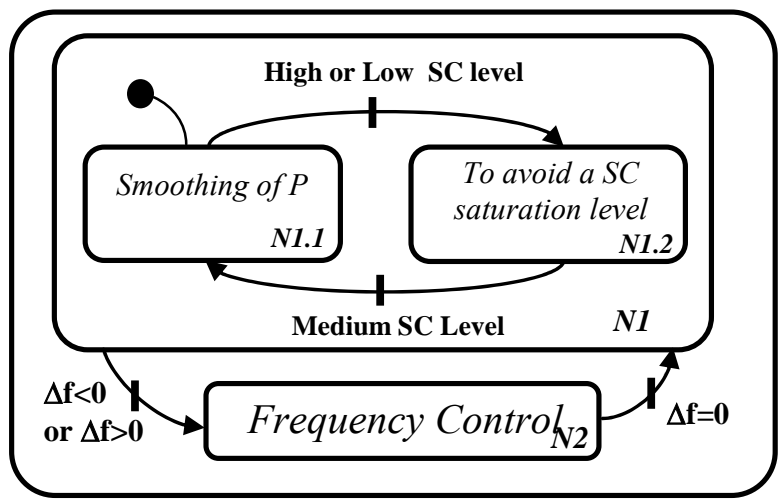

Fig. 7. Chart representation of the different operating modes

The chart representation of operating modes in Fig.7 shows two levels.

- The level $N 1$ explains the power smoothing. There have two objectives: to smooth the power (N1.1) and to avoid the low and high saturation states of supercapacitors (N1.2).

- The level N2 is the frequency control. We associate fuzzy logic rules for each operating mode. Fuzzy logic allows to work in several levels in the same time. 


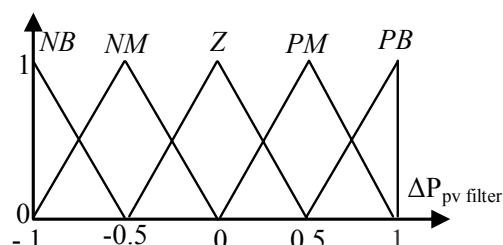

a)

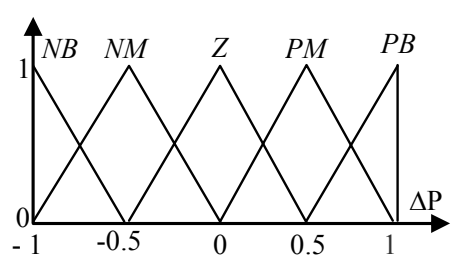

d)

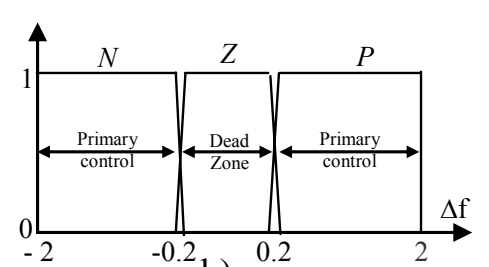

b)

$N$ : Negative

$Z:$ Zero

$P$ : Positive

$M:$ Medium

B : Big

$S:$ Small

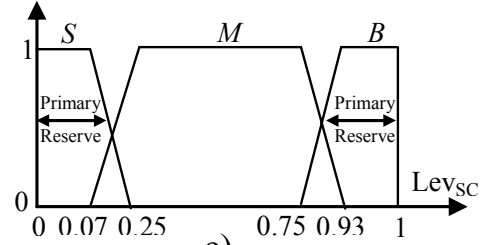

c)

NB: Negative Big

PB: Positive Big

PM: Positive Medium

NM: Negative Medium

VB : Very Big

Fig. 8: Fuzzyfication, membership functions of: a) $\Delta \mathrm{P}_{\mathrm{PV}}$ filtered, b) Frequency variations, c) SC Level, d) Power variations

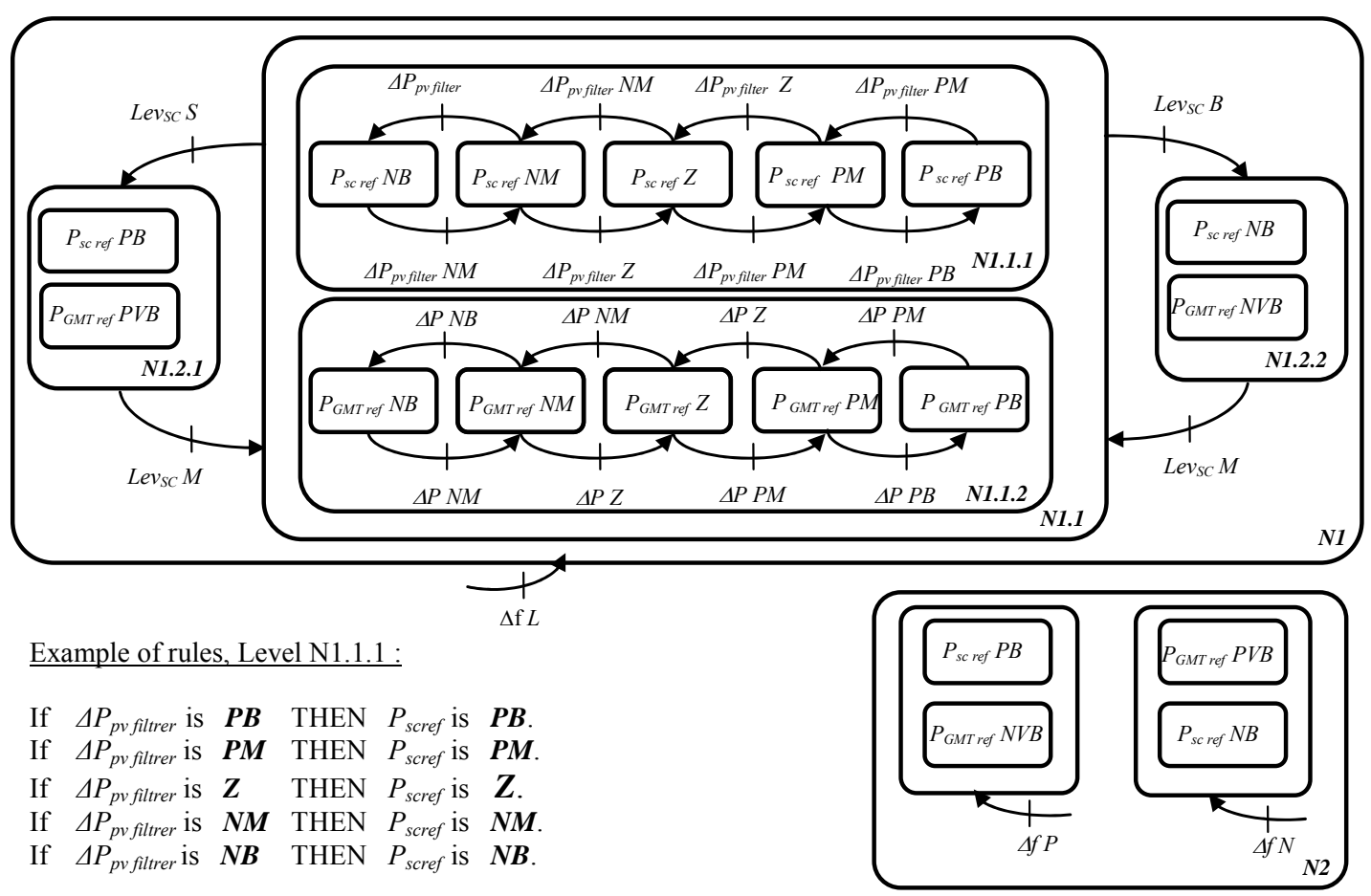

Fig. 9: Inference rules Level N1: Smoothing of power, Level N2: Frequency control

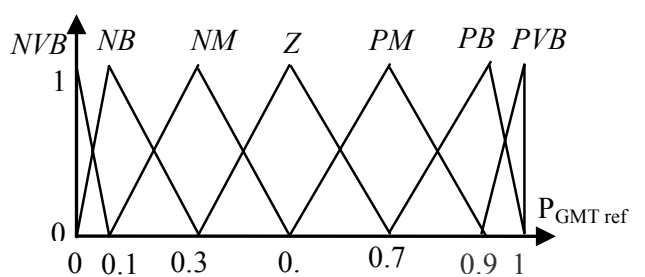

a)

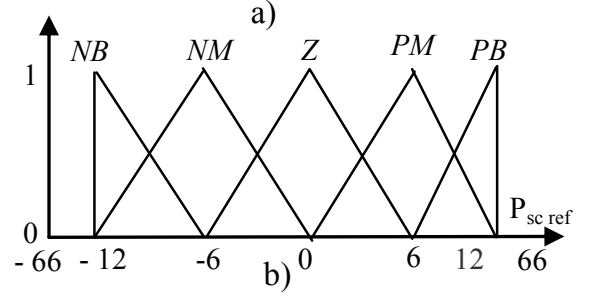

Fig10. Defuzzyfication, membership functions of: a) GMT Power b) SC power

\section{Fuzzy Logic Supervisor}

Three steps characterize a fuzzy logic supervisor:

- Fuzzyfication (Fig.8), which consists to convert the inputs with membership functions.

- Inference (Fig.9), which consists to define the rules.

- Defuzzyfication (Fig.10), which consists to convert the outputs with membership functions.

Fig.9 shows a chart representation to deduce more easily the fuzzy rules. Two levels are considered. Level N1 allows to provide the reference power and to avoid a SC saturation level. When the SC level is not high or low then supercapacitors compensate the fast fluctuations of 
power (N.1.1.1) and GMT compensates the low fluctuations of power (N.1.1.2).

Level $N 2$ concerns the frequency control. When there is a frequency variation then the GMT must increase or decrease this generated power. At the same time SC participate to the frequency control.

\section{Simulations}

The studied network shown in Fig.1. The simulation time is 3 hours with a reference power for the hybrid DG changing each hour $P_{H G}$ ref $=\{24 ; 30 ; 26\} \mathrm{kW}$. Each hour a $40 \mathrm{~kW}$ load is disconnected for $400 \mathrm{~s}$. The grid manager sends a secondary load frequency level (secondary frequency control) 200s after the load disconnection, which is equivalent to a reference power of $-2 \mathrm{~kW}$ for 200s. The fuzzy logic supervision, represented in continuous dark curves, is compared to the basic supervision strategy, represented in dotted light curves. Fig. 11 shows the results of the total simulation, while the figure 12 focus on the first hour of simulation. Fig.11a shows the photovoltaic power, figure $11 \mathrm{~b}$. Fig. 11 shows the photovoltaic power (fig 11.a), the GMT power (Fig.11b), the SC power (Fig.11c), the hybrid power (Fig.11d), the SC level (Fig.11d) and the grid frequency (Fig.11d).

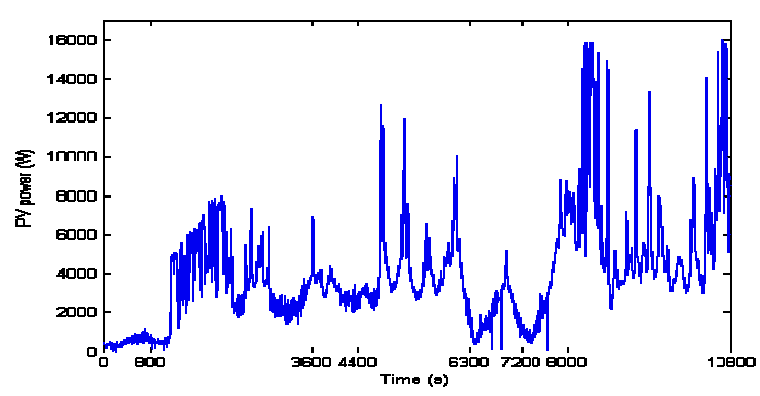

(a)

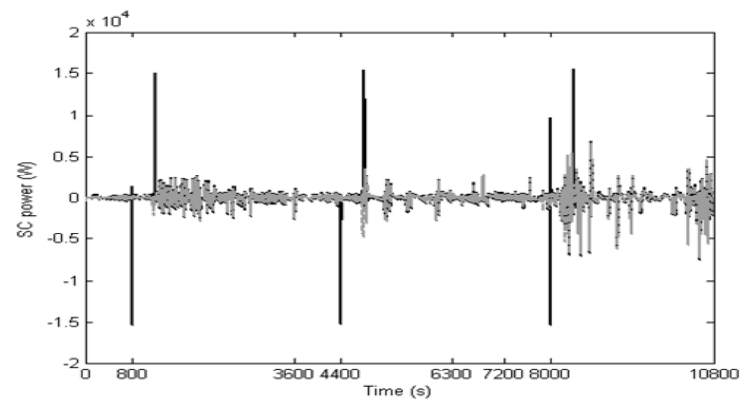

(c)

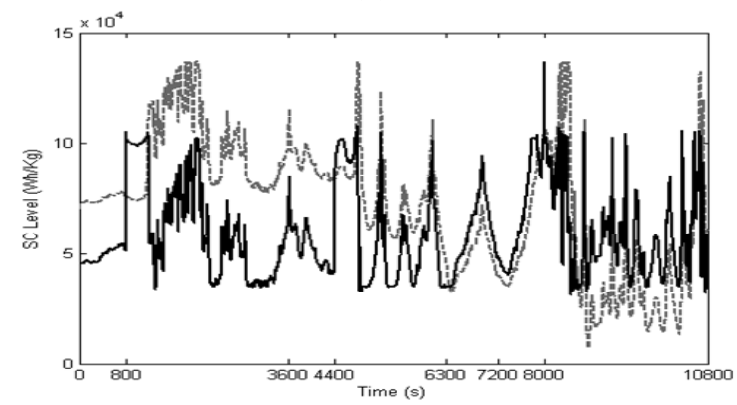

(e)
For a photovoltaic power (Fig.11a), we note that with the GMT (Fig.11b) and the supercapacitors (Fig11.c and 11.e) the hybrid DG source provides the reference power (Fig.11d) on the micro, grid and so the power requested by the grid manager on the grid. Thus the frequency is stabilized and improved contrary to the basic strategy (Fig11.f). We can note that for each reference power the primary and secondary frequency control are performed. The energy level of the supercapacitors is, except at the time of the frequency control, never in saturation state in the case of fuzzy logic supervisor, thus the HG provides a more regular power reserve to contribute to primary frequency control. The small droop of power observed at $t=6300 \mathrm{~s}$ is due to the photovoltaic power being low; the GMT is in high saturation and cannot provide the power requested any more.

Fig.12 shows the grid frequency (fig 12.a), the GMT power (Fig.21b), the SC power and level (Fig.12c), the hybrid power (Fig.12d).

When the load is disconnected at $t=800 \mathrm{~s}$, the SC and GMT power decrease (Fig.12b and 12c) to limit the frequency deviation. The peak of frequency $50.97 \mathrm{~Hz}$ is decreased with $50.60 \mathrm{~Hz}$ (Fig.12a).

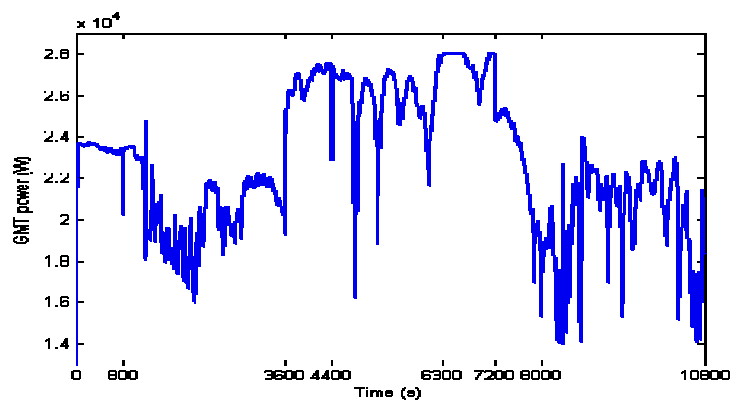

(b)

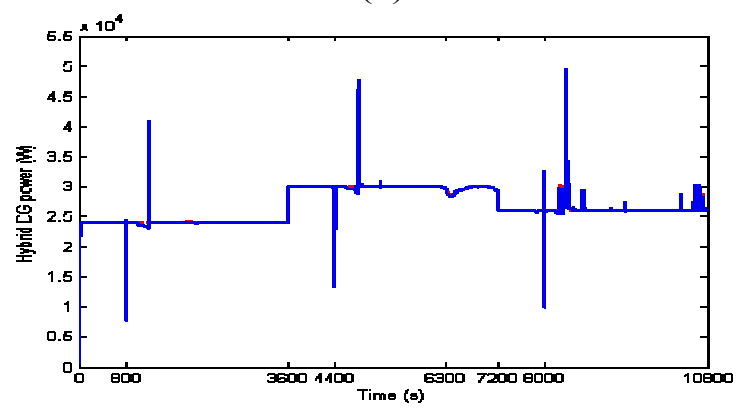

(d)

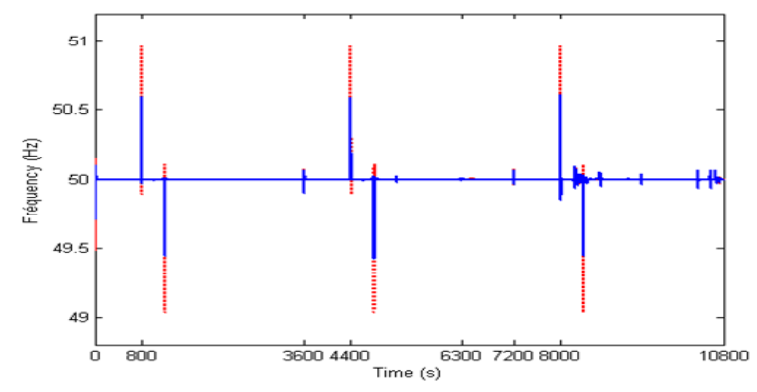

(f)

Fig. 11. a) PV power b) GMT power c) SC power and level d) Hybrid DG poxer e) SC level f) frequency 


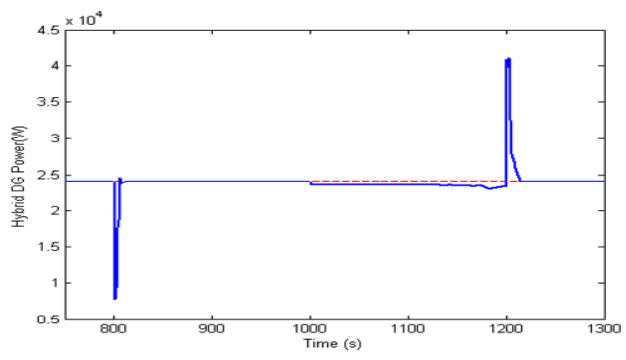

(a)

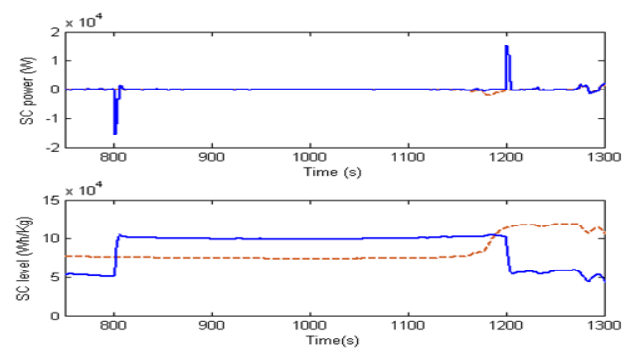

(c)

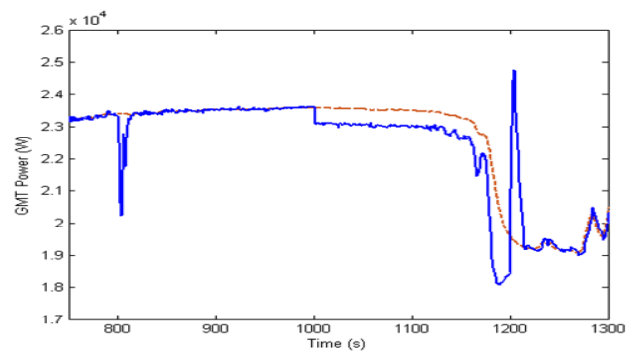

(b)

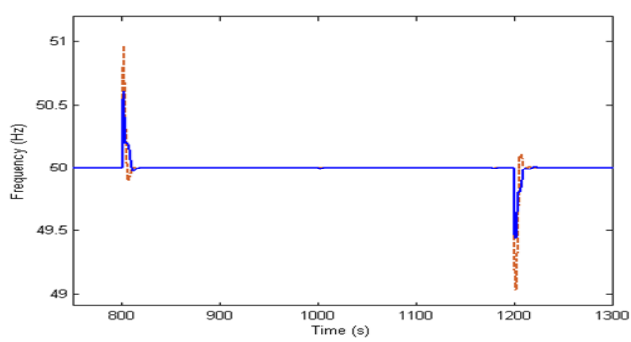

(d)

Fig. 12. a) PV power b) GMT power c) SC power and level d) Hybrid DG poxer

In a second step the inertia of the revolving machines allows it to stabilize the frequency. At $t=1000 \mathrm{~s}$ the grid manager sends a secondary load frequency level and the MTG power increase.

In the same way, when a load is connected the $\mathrm{SC}$ and GMT power increase (Fig.12b and 12c). The peak of frequency is decreased from $49.04 \mathrm{~Hz}$ to $49.44 \mathrm{~Hz}$. The power delivered by the hybrid source corresponds to the reference power when the frequency is equal to $50 \mathrm{~Hz}$.

\section{Conclusion}

A supervision methodology for a hybrid generation system has been developed in this paper. This strategy enables to improve the grid insertion of renewable distributed generator (DG) by associating them a short term storage system and available decentralized generator (FDG). A proposed fuzzy logic supervisor allows to maintain a reference power and to contribute to frequency control with a hybrid generator (HG) based on photovoltaic arrays, gas micro turbine and supercapacitors. The proposed fuzzy logic supervisor is compared with a basic supervision strategy The FLS gives a better storage management by reducing high and low saturation state, thus the HG can provide a more regular power reserve to contribute to the primary frequency control. A simulation with the help of Matlab Simulink shows that the Hybrid Generator can follow a reference power and participe to the frequency control.

\section{References}

[1] R. Lasseter, A. Akhil, C. Marnay, J. Stephens, J. Dagle, R. Guttromson, A. Meliopoulos, R. Yinger, and J. Eto, "White Paper on Integration of Distributed Energy Resources. The CERTS MicroGrid Concept,", CA, Tech. Rep. LBNL-50 829, 2002.

[2] D. Kondoleon, L. Ten-Hope, T. Surles, R.L. Therkelsen, "Integration of distributed energy". The CERTS micro grid concept, California Energy Commission, 2003.

[3] S. Abu-Sharkh, "Can micro grids make a major contribution to UK energy supply?", Renewable and Sustainable Energy Reviews 10. pp. 78-127, 2006.
[4] S. Wijnbergen,S.W.H de Haan and J.G. Slootweg "A system for dispersed generator participation in Voltage control and Primary frequency control", IEEE $36^{\text {th }}$ Power Electronics Conference, 2005.

[5] J. Morren, S W.H. de Haan, J.A. Ferreira, "Contribution of DG units to primary frequency control" in Future Power Systems 2005, Amsterdam, the Netherlands, November 16$18,2005$.

[6] V. Courtecuisse, M. El Mokadem, B. Robyns, B. François, M. Petit, J. Deuse, "Association of wind turbine based dispersed generators and storage systems to participate in primary frequency control", EPE $2007-12^{\text {th }}$ European Conference on Power Electronics Conference, Aalborg, Denmark, 2-5 septembre, Denmark, CDROM

[7] P. Kundur, Power "System stability and control", Power Research Institute, ISBN -07-035958-X, McGraw-Hill, Inc., 1993.

[8] Ph. Degobert, S. Kreuawan, X. Guillaud, "Micro-grid powered by photovoltaic and micro turbine", International Conference on Renewable Energy and Power Quality (ICREPQ'06) April 2006, Palma de Mallorca, Spain, CDROM

[9] P. Li, Ph. Degobert, B. Robyns, B. François, "Modelling and Control of a Micro Turbine Generator by Using Causal Ordering Graph", Computational Engineering in Systems Applications: CESA, Beijing, China, 4-6 Octobre, 2006, CDROM.

[10]RTE, France, Mèmento de la sûreté électrique 2004. [online] Available : http://www.rte-france.com.

[11] Y. G.Rebours, D. S.Kirschen, M. Trotignon and S. Rossignol, „A survey of Frequency and Voltage Control Ancillary Service-Part I: Technical Feature", IEEE transaction on power system, Vol.22, $\mathrm{N}^{\circ} 1$., February 2007, pp :350-357.

[12]V. Courtecuisse, B. Robyns, S. Plumel, B. Francois and J. Deuse, "Capacity of a variable speed turbine to participate in primary frequency control", Sixth international workshop on large-scale integration of wind power and transmission networks for offshore wind farms, Delft, October 26-27, 2006, CDROM.

[13]G. Cimuca, C. Saudemont, B. Robyns, M. Radulescu "Control and performance evaluation of a flywheel energy storage system associated to a variable speed wind generator", IEEE Transactions on Industrial Electronics. Vol. 53, No. 4, June 2006, pp:1074 - 1085. 